\title{
Method For Artefact Detection And Removal In Heart Rate Signals Measured During Physical Exercise
}

\author{
Pieter Joosen ${ }^{1,2}$, Vasileios Exadaktylos ${ }^{1}$, Joachim Taelman ${ }^{2}$, Jean-Marie Aerts ${ }^{1}$ and Daniël \\ Berckmans ${ }^{1}$ \\ ${ }^{1}$ Division Measure, Model \& Manage Bioresponses, KU Leuven, Kasteelpark Arenberg 30, Leuven, Belgium \\ ${ }^{2}$ BioRICS NV, Technologielaan 3, Heverlee, Belgium \\ \{pieter.joosen, joachim.taelman\}@biorics.com, \{Vasileios.Exadaktylos, Jean-Marie.Aerts, \\ Daniel.Berckmans\}@biw.kuleuven.be
}

Keywords: Heart rate, artefact detection, artefact removal, pre-processing.

\begin{abstract}
Monitoring the training of athletes is essential for success in sports. Information that is derived from measured data during the training is only reliable if the quality of the data is high. Therefore artefact detection and removal are essential. In this paper typical artefacts in heart rate measurements during football field-trainings are described. An algorithm to automatically detect artefacts and an algorithm to pre-process them are also presented. The results show that with the proposed pre-processing method the percentage of artefacts can be significantly $(\mathrm{p}<0.01)$ reduced from 9.0 to $4.4 \%$. This corresponds to a total period of 10 hours of unreliable data on a total of 126 hours that have been taken out. As a result, more high quality data is available for monitoring training. The developed methods are generic and can be used in many applications where accurate heart rate monitoring is crucial.
\end{abstract}

\section{INTRODUCTION}

Monitoring the training of athletes is essential for their success. Training programs can only be optimized when accurate information about the training and its effect on the athlete is available. Today more attempts are being made to gather quantitative information about trainings and their effect by using modern technology.

Training is affected by the type, duration, frequency and intensity of exercises (Impellizzeri et al, 2005). The concept of the training impulse (TRIMP, Banister, 1991) integrates these components in a single term, based on heart rate measurements during training. Other research has shown that quantification of the dynamic relationship between physical activity and heart rate contains information about the physical condition of athletes (Lefever et al., 2012).

An important limitation of methods based on measuring field data and in particular heart rate is the quality of the measured signal. During intense training, technical failure of the measuring equipment is often causing loss of data and/or artefacts. As a consequence information that is derived from heart rate may also be incorrect.
Therefore monitoring training with heart rate is only reliable when artefacts can be detected and removed. In general it has no meaning to use the limited energy available in wearable technology to send low quality data higher up for analysis.

Heart rate is often derived from R-R interval time series from electrocardiographic (ECG) recordings. These R-R interval time series can contain artefacts of either physiological or technical origin (Peltola, 2012). Many algorithms for correcting artefacts in R-R intervals have been developed, such as the $20 \%$ filter (Kleiger et al., 1987), deletion of the false R-R intervals, etc. However, if only heart rate is available instead of the $\mathrm{R}-\mathrm{R}$ intervals, other approaches are needed.

Therefore typical artefacts in heart rate data, measured during football field-trainings, are described in this paper. Next an algorithm to automatically detect these artefacts is presented. If artefacts are present in the data, these can be processed with the presented pre-processing algorithm. Finally the influence of the preprocessing method on the artefacts is investigated.

Please cite as:

P. Joosen, V. Exadaktylos, J. Taelman, J.-M. Aerts and D. Berckmans. Method for artefact detection and removal in heart rate signals measured during physical exercise. In: Proceedings of the International Congress on Cardiovascular Technologies - CARDIOTECHNIX 2013, Villamoura, Portugal, 19-21 September 2013. 


\section{METHODS}

\subsection{Data Acquisition}

The data for this study are part of a larger project that studies the dynamic interactions between velocity and heart rate of football players during field-trainings. For this analysis high quality heart rate measurement is essential.

Fourteen players (age: 17.6 \pm 0.7 year) are monitored for a total of 127 field-trainings with the Inmotio LPM system (Inmotio Object Tracking BV, the Netherlands) and Hosand Heart Rate module (Hosand Technology, Italy) with Polar heart rate belt (Polar Electro, OY, Finland). Velocity and heart rate are sampled at $5 \mathrm{~Hz}$. Field-trainings consist of different types of exercises (match, shooting, sprints, running, etc.) and have a duration of $1 \mathrm{~h} \pm 30 \mathrm{~min}$. In total 126 hours of measurements are available.

\subsection{Artefacts}

In the measured heart rate data, three typical artefacts can be distinguished. These are defined in the first sub-section. An algorithm to automatically detect each artefact type is presented in the following sub-section.

\subsubsection{Definition of Artefact Types}

Reliable heart rate measurements during football trainings normally show values lower than $230 \mathrm{bpm}$ and higher than $40 \mathrm{bpm}$ (Atwal, 2002). Periods when heart rate measures are outside these bounds, are defined as type I artefacts (Figure 1, bottom left).

During exercise the heart responds to the physical activity, changing the heart rate to fulfil physiological requirements. Measured values of heart rates changing faster than physiologically possible, may occur due to technical failures. These high-frequency errors are defined as type II artefacts (Figure 1, bottom centre).

The heart is influenced by many factors (Ryan, 1994). Therefore measured values of heart rate that are constant or changes at a constant pace for a longer duration are not plausible. These are defined as type III artefacts (Figure 1, bottom right).

\subsubsection{Detection Algorithm}

Each type of artefact can be automatically detected using specific criteria.

Detection of type I artefacts is most straightforward. Whenever heart rate exceeds 230 bpm or drops below $40 \mathrm{bpm}$ during a certain time, the algorithm detects this period as an artefact of type I (Figure 2, second plot).

To detect type II artefacts, the heart rate change per sample is computed. This is subsequently filtered with a moving window average filter (window $0.8 \mathrm{~s}$ ) to take out high-frequent changes. This signal is used to threshold for artefacts: if the filtered heart rate change per second exceeds 15 $\mathrm{bpm} / \mathrm{s}$, a type II artefact is detected (Figure 2, third plot).
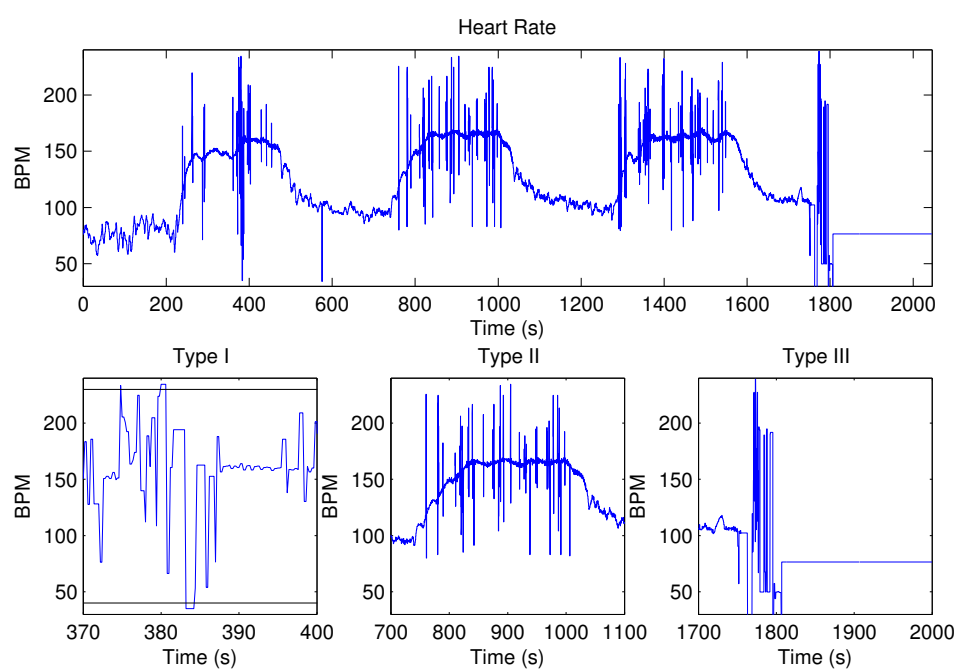

Figure 1: Example of heart rate containing the three types of artefacts. 
Type III artefacts are characterized by a constant heart rate and constant heart rate changes. Thus the variation in heart rate changes is low at these instances. Therefore the moving standard deviation of the filtered heart rate change is computed (window $2 \mathrm{~s}$ ). If the variation is smaller than 0.01 $\mathrm{bpm} / \mathrm{s}$, the algorithm detects a type III artefact (Figure 2, fourth plot).

The thresholds that are used for the detection of type II and III artefacts are selected by trial and error.

\subsubsection{Performance Measures}

The percentage of time that one training session contains artefacts is computed for all trainings. This is done for each type of artefact separately, as well as for all types combined. These measures are weighted with the duration of the training: the largest weight is 1 and corresponds with a training duration of $2 \mathrm{~h}$.

\subsection{Pre-processing Algorithm}

In the project, for which this data is acquired, a high quality and reliable heart rate signal of $1 \mathrm{~Hz}$ is required. The first step in the pre-processing algorithm therefore would be to low-pass filter the 5 $\mathrm{Hz}$ heart rate signal to remove high-frequent information. However, this filtering introduces large errors in periods where artefacts are present. Therefore, artefacts are first removed from the heart rate by linear interpolation (Figure 3, second plot). During our measurements the median duration of type I, II and III artefacts were 4.1, 1.7 and 14.8 seconds respectively. Therefore linear interpolation produced adequate results.

Next a low-pass Butterworth filter with cut-off frequency of $1 \mathrm{~Hz}$ is applied on this signal (Figure 3, third plot).

Finally heart rate during artefacts is replaced with cubic interpolation, to take into account the non-linear characteristics of heart rate (Figure 3, fourth plot).

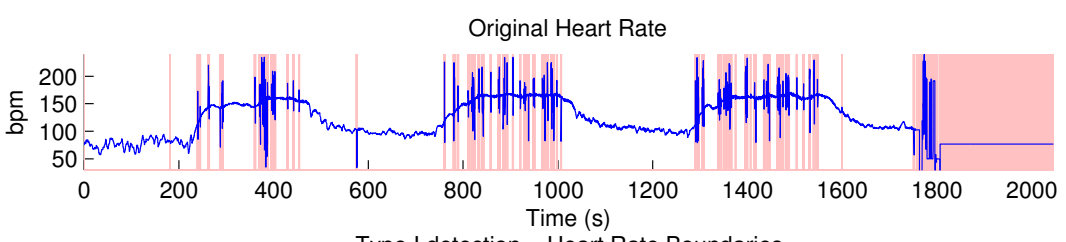

Type I detection - Heart Rate Boundaries

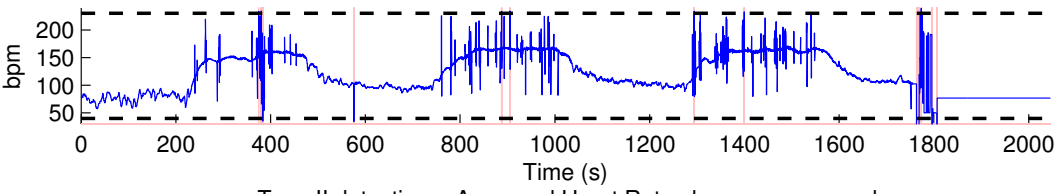

Type II detection - Averaged Heart Rate change per second

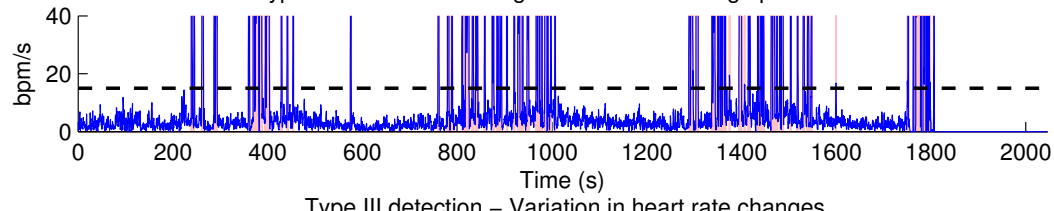

Type III detection - Variation in heart rate changes

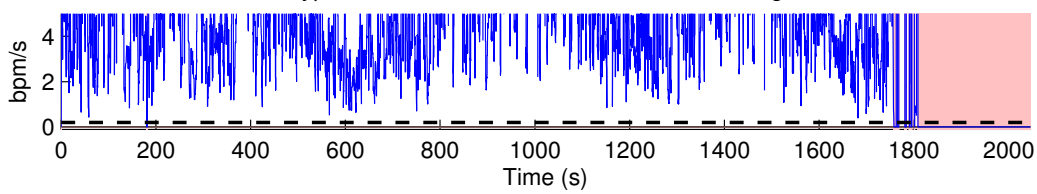

Figure 2: Artefact detection algorithm steps. The shaded areas represent detected artefacts. The dashed horizontal lines represent the thresholds used in the algorithm. 

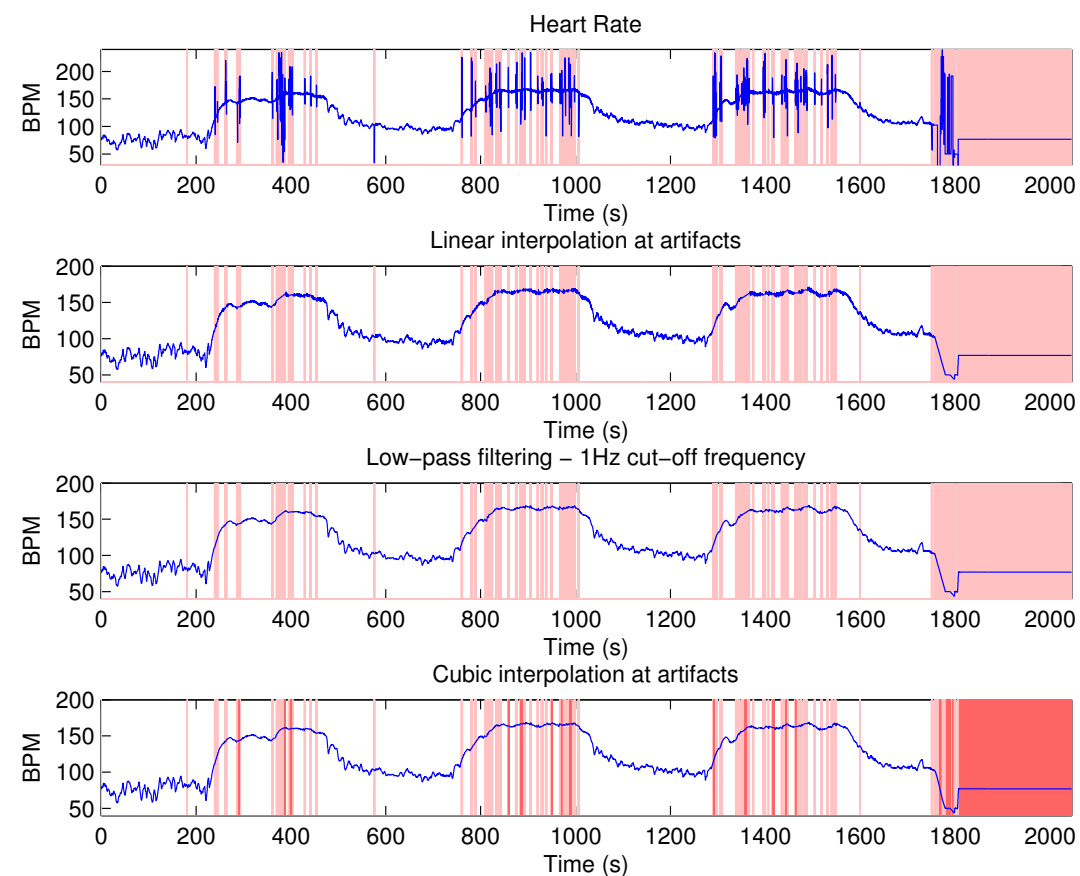

Figure 3: Heart rate pre-processing algorithm steps. The light shaded areas represent the detected artefacts before preprocessing, the dark shaded areas after pre-processing.

\subsection{Statistical Analysis}

The performance measures are calculated before and after pre-processing. The effect of pre-processing on the different types of artefacts can thus be investigated.

Normality of each group is tested with a Lilliefors test. The null-hypothesis (data is distributed normally) was rejected for each group $(\mathrm{p}<0.001)$. Since comparisons are made between two paired, non-parametric groups, the Wilcoxon test is used.

Results and Discussion

Table 1 reveals that most of the artefacts in this dataset are of type III; the median of type I and II artefacts are only $0.1 \%$ and $0.9 \%$ respectively, while this is $6.7 \%$ for type III. The median of the total percentage of artefacts is $9.0 \%$, which corresponds with 11 minutes of unreliable data per training. In total 37 hours of artefacts are present in the data.

Another interesting result is that the total percentage of artefacts is reduced significantly $(\mathrm{p}<0.01)$ after pre-processing. The total period of data containing artefacts is reduced with more than 10 hours. Even more, type I and II artefacts are almost completely removed from the raw data. The pre-processing algorithm presented in this paper is thus able to reduce type I and II artefacts significantly $(\mathrm{p}<0.01)$.

Type III artefacts on the other hand are still present after pre-processing, although they are decreased from 6.7 to $4.2 \%$. In this case more advanced methods are required.

\section{CONCLUSIONS}

In this paper an artefact detection algorithm for heart rate is presented, which is able to automatically detect three types of artefacts; physiologically not meaningful heart rates (type I), heart rate changes that are too fast (type II) and changes that show too little variation (type III).

The pre-processing algorithm could not reduce type III artefacts. More advanced techniques are needed here to recover this data. Nevertheless periods in heart rate still containing errors can be detected automatically. In further analysis this information can be taken into account.

Table 1: Median and interquartile range, ${ }^{\mathbf{a}}$ shows statistical significance $(p<0.01)$ between group before and group after pre-processing. 


\begin{tabular}{|cc|c|c|}
\hline \multicolumn{2}{|c|}{ Artefacts } & $\begin{array}{c}\text { Before Pre- } \\
\text { processing }\end{array}$ & $\begin{array}{c}\text { After Pre- } \\
\text { processing }\end{array}$ \\
\hline Total & $(\%)$ & $9.0_{+9.8}^{-6.0} \mathrm{a}$ & $4.4_{+8.1}^{-4.1}$ \\
\hline Type I & $(\%)$ & $0.1_{+1.4}^{-0.1} \mathrm{a}$ & $0_{+0.02}^{-0}$ \\
\hline Type II & $(\%)$ & $0.9_{+1.0}^{-0.5} \mathrm{a}$ & $0.04_{+0.13}^{-0.03}$ \\
\hline Type III & $(\%)$ & $6.7_{+10.4}^{-6.4}$ & $4.2_{+8.2}^{-4.0}$ \\
\hline
\end{tabular}

It is also shown that with pre-processing the total percentage of artefacts can be significantly reduced. Typical artefacts (type I and II) in heart rate can even be almost completely removed from raw data.

As a result, more high quality data is available for analysis and for a total period of 10 hours bad data have been taken out. The developed methods are generic and can be used in many applications where accurate heart rate monitoring is crucial.

\section{REFERENCES}

Atwal, S, Porter, J, MacDonald, P, 2002. Cardiovascular effects of strenuous exercise in adult recreational hockey: the hockey heart study. In CANADIAN MEDICAL ASSOCIATION JOURNAL, Vol. 166, No. 3, pp. 303-307.

Banister, E, 1991. Modeling Elite Athletic Performance. In MacDougal, J, Wenger, $\mathrm{H}$, Green, $\mathrm{H}$, PHYSIOLOGICAL TESTING OF ELITE ATHLETES, $2^{\text {nd }}$ edition, pp. 403-424.

Impellizzeri , F, Rampinini, E, Marcora, S, 2005. Physiological assessment of aerobic training in soccer. In JOURNAL OF SPORTS SCIENCE, vol. 23, no. 6, pp. 583-592

Kleiger, R., Miller, J., Bigger, Jr. I., Moss, A., 1987. Decreased heart rate variability in association with increased mortality after acute myocardial infarction. In THE AMERICAN JOURNAL OF CARDIOLOGY, vol. 59 , no. 4 , pp. 256-262

Lefever, J, Berckmans, D, Aerts, J-M, 2012. Time-variant modelling of heart rate responses to exercise intensity during road cycling. In EUROPEAN JOURNAL OF SPORT SCIENCES, vol. in press. Available from Taylor \& Francis.

Peltola, M., 2013. Role of editing of R-R intervals in the analysis of heart rate variability. In FRONTIERS IN PHYSIOLOGY, vol. 3, no. 148, pp. 1-10

Ryan, S, Goldberger, A, Pincus, S, Mietus, J, Lipsitz, L, 1994. Gender- and age-related difference in heart rate dynamics. In JOURNAL OF THE AMERICAN COLLEGE OF CARDIOLOGY, Vol.24, No. 7. 